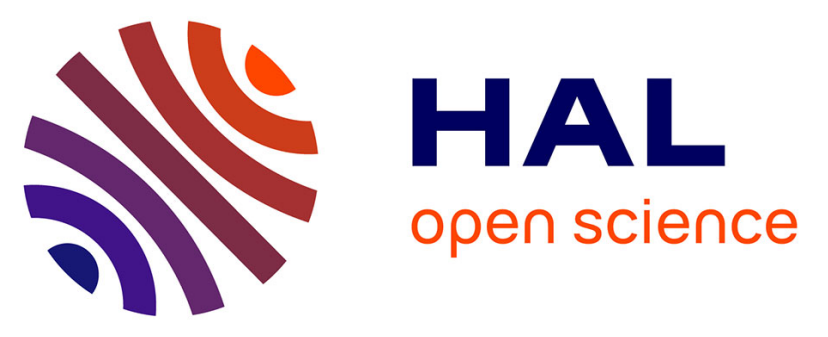

\title{
Experimental Dynamic Identification of a Fully Parallel Robot
}

\author{
Oscar Andrès Vivas, Philippe Poignet, Frédéric Marquet, François Pierrot, \\ Maxime Gautier
}

\section{- To cite this version:}

Oscar Andrès Vivas, Philippe Poignet, Frédéric Marquet, François Pierrot, Maxime Gautier. Experimental Dynamic Identification of a Fully Parallel Robot. ICRA: International Conference on Robotics and Automation, Sep 2003, Taipei, Taiwan. pp.3278-3284. lirmm-00269510

\section{HAL Id: lirmm-00269510 \\ https://hal-lirmm.ccsd.cnrs.fr/lirmm-00269510}

Submitted on 6 Jul 2016

HAL is a multi-disciplinary open access archive for the deposit and dissemination of scientific research documents, whether they are published or not. The documents may come from teaching and research institutions in France or abroad, or from public or private research centers.
L'archive ouverte pluridisciplinaire HAL, est destinée au dépôt et à la diffusion de documents scientifiques de niveau recherche, publiés ou non, émanant des établissements d'enseignement et de recherche français ou étrangers, des laboratoires publics ou privés. 


\title{
Experimental dynamic identification of a fully parallel robot
}

\author{
Andrès Vivas* Philippe Poignet* Frédéric Marquet* François Pierrot* Maxime Gautier§ \\ *Laboratoire d'Informatique, Robotique et Microélectronique de Montpellier - UMR CNRS 5506, 161 Rue Ada, 34392 \\ Montpellier cedex 5, France \\ §Institut de Recherche en Communication et Cybernétique de Nantes, UMR CNRS 6597, 1 rue de la Noë, 44321 \\ Nantes cedex 03, France \\ \{vivas, poignet\}@lirmm.fr
}

\begin{abstract}
This paper deals with the experimental identification of the dynamic parameters of parallel machines. The dynamic parameters are estimated by using the weighted least squares solution of an over determined linear system obtained from the sampling of the dynamic model along a closed loop exciting trajectory. Experimental results are exhibited for the $\mathrm{H} 4$ robot, a fully parallel structure providing 3 degrees of freedom (dof) in translation and 1 dof in rotation. A comparative study is performed depending on the available measurements i.e. different sensor locations (motor, end effector).
\end{abstract}

\section{INTRODUCTION}

After the works in parallel mechanisms introduced by Gough [1] or Steward [2], Clavel [3] proposed the Delta structure, a parallel robot dedicated to high-speed applications. In the same way, the "hexapod" [4], [5] has been used intensively in industry. This is due to the exceptional simplicity of the Delta 3-dof solution and the enormous research effort dedicated to the "hexapod". Many alternate designs have been proposed like the HexaM [6], which is an evolution of the Hexa robot [7]. For most pickand-place applications, at least four dof are required (3 translations and 1 rotation to arrange the carried object in its final location). For the Delta robot, this is achieved thanks to an additional link between the base and the gripper, but it seems not to be as efficient as a parallel arrangement. On the other hand, 6-dof fully-parallel machines currently used in machining suffer from their complexity (they need at least 6 motors while the cutting process requires only 5 controlled axis plus the spindle rotation) and from their limited tilting angle. As an intermediate solution to these drawbacks, a 4dof parallel mechanism - the $\mathrm{H} 4$ robot - have been proposed [8], [9]. Fig. 1 shows a photography of the H4 parallel robot. This machine is based on 4 independent active chains between the base and the nacelle; each chain is actuated by a brushless direct drive motor fixed on the base and equipped with an incremental position encoder. Thanks to its design, the mechanism is able to provide high performances. In order to achieve high speed and acceleration for pick-and-place applications or precise motion in machining tasks, an accurate dynamic modeling is required to increase the quality of their simulation in order to improve their design and to compute advanced model based robust controllers such as moving horizon control schemes. However the first difficulty is to estimate the physical parameters (mass, inertia and frictions), especially when the only available measurements are given by the incremental sensors located on the actuators.

Therefore, in this paper, we focus on the estimation of the dynamic parameters of the rigid multi body model. The parameters are estimated by a classical technique of weighted least squares [11], [14]. We mainly discuss two identification results depending on the available measurements. We compare the influence of the sensor locations on the estimation results of physical parameters: i) first, the nacelle acceleration is estimated through the computation of the kinematic model and its derivative ii) secondly, additional sensors (rotation and 3-axis acceleration sensors) located on the end effector provide further measurements.

The paper is organized as follows : Section 2 is dedicated to the geometric, kinematic and dynamic modelling. Section 3 recalls the basis of the identification method. Section 4 exhibits and discuss experimental identification results of the H4 robot. Finally, conclusions are given in section 5 .

\section{MODELING}

\section{A. Geometric and kinematic modelling}

The Jacobian matrix and the forward geometric model are needed to compute the dynamic model (see section 2.2). Therefore we briefly present the way of computing the different relationship necessary to obtain these model and matrix. The design parameters of the robot are described on Fig. 2 where the following parameters have been chosen:

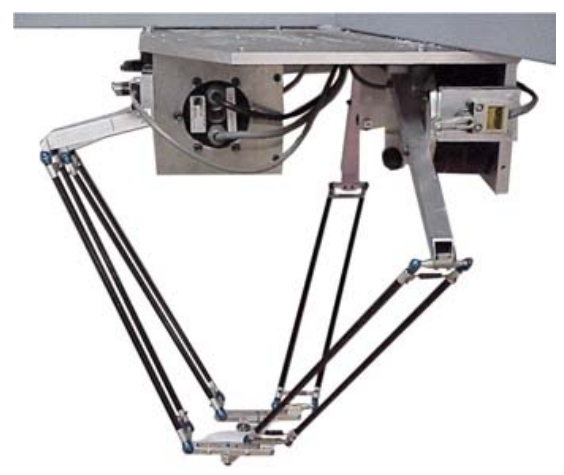

Fig. $1 \mathrm{H} 4$ robot 
$\alpha_{1}=0 ; \alpha_{2}=\pi ; \alpha_{3}=3 \pi / 2 ; \alpha_{4}=3 \pi / 2$

$\boldsymbol{u}_{1}=\boldsymbol{u}_{y} ; \boldsymbol{u}_{2}=-\boldsymbol{u}_{y} ; \boldsymbol{u}_{3}=\boldsymbol{u}_{x} ; \boldsymbol{u}_{4}=\boldsymbol{u}_{x}$

The angles $\alpha_{i}$ describe the position of the four motors, $L$ is the length of arms, $l$ is the length of the forearms, $\theta$ the nacelle's angle, and $d$ and $h$ are the half lengths of the "H" forming the nacelle. $\boldsymbol{O}$ is the origin of the base frame and $D$ is the origin of the nacelle frame. $R$ gives the motor's position. The $\boldsymbol{A}_{\boldsymbol{i}} \boldsymbol{B}_{\boldsymbol{i}}$ segments represent the arms of the robot and $\boldsymbol{P}_{\boldsymbol{i}} \boldsymbol{B}_{\boldsymbol{i}}$ the forearms segments. The joint positions are represented by $q_{i}$.

To obtain the geometric model, it is necessary to express the different points of the mechanical system with respect to the origin $\boldsymbol{O}$. The origin is fixed in the middle of the nacelle with the coordinates $(x, y, z)$. In the Cartesian space, the end effector position is given by $(x, y, z, \theta)$.

$\boldsymbol{O D}=\left[\begin{array}{lll}x & y & z\end{array}\right]^{T}$

The vector that joins the absolute origin $O$ and all of the forearms to the nacelle is:

$$
\boldsymbol{O} \boldsymbol{A}_{\boldsymbol{i}}=\boldsymbol{O D}+\boldsymbol{D} \boldsymbol{A}_{\boldsymbol{i}}=\left[\begin{array}{l}
x \\
y \\
z
\end{array}\right]+\boldsymbol{D} \boldsymbol{A}_{\boldsymbol{i}}
$$

The $\boldsymbol{D} \boldsymbol{A}_{\boldsymbol{i}}$ segments can be expressed as:

$$
\begin{aligned}
\boldsymbol{D}_{\boldsymbol{1}} & =\left[\begin{array}{c}
h \cos \theta \\
h \sin \theta+d \\
0
\end{array}\right] ; \boldsymbol{D A}_{\mathbf{2}}=\left[\begin{array}{c}
-h \cos \theta \\
-h \sin \theta+d \\
0
\end{array}\right] \\
\boldsymbol{D A}_{3} & =\left[\begin{array}{c}
-h \cos \theta \\
-h \sin \theta-d \\
0
\end{array}\right] ; \boldsymbol{D A}_{4}=\left[\begin{array}{c}
h \cos \theta \\
h \sin \theta-d \\
0
\end{array}\right]
\end{aligned}
$$

Moreover, the vector that links the absolute origin and all of the arms to the forearms is:

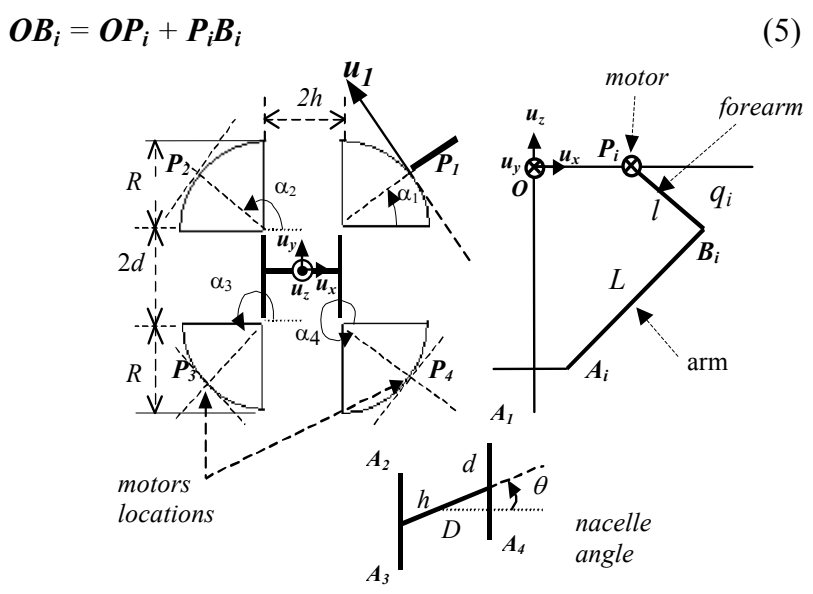

Fig 2. Design parameters with:

$$
\boldsymbol{P}_{\boldsymbol{i}} \boldsymbol{B}_{\boldsymbol{i}}=\left[\begin{array}{c}
l \cos q i \cos \alpha i \\
l \cos q i \sin \alpha i \\
-l \sin q i
\end{array}\right]
$$

and actuator locations are:

$$
\begin{gathered}
\boldsymbol{O P}_{1}=\left[\begin{array}{c}
h+R \cos \alpha_{1} \\
d+R \sin \alpha_{1} \\
0
\end{array}\right] ; \boldsymbol{O} \boldsymbol{P}_{2}=\left[\begin{array}{c}
-h+R \cos \alpha_{2} \\
d+R \sin \alpha_{2} \\
0
\end{array}\right] \\
\boldsymbol{O P}_{3}=\left[\begin{array}{c}
-h+R \cos \alpha_{3} \\
-d+R \sin \alpha_{3} \\
0
\end{array}\right] ; \boldsymbol{O} \boldsymbol{P}_{4}=\left[\begin{array}{c}
h+R \cos \alpha_{4} \\
-d+R \sin \alpha_{4} \\
0
\end{array}\right]
\end{gathered}
$$

Finally, arms coordinates are given by:

$A_{i} B_{i}=A_{i} O+O B_{i}$

The analytical forward position relationship is difficult to compute. Up to now, the simplest model we've got is a $8^{\text {th }}$ degree polynomial equation. The forward model is then computed iteratively using the classical formula:

$\boldsymbol{x}_{n+1}=\boldsymbol{x}_{n}+\boldsymbol{J}\left(\boldsymbol{x}_{n}, \boldsymbol{q}_{n}\right)\left[\boldsymbol{q}-\boldsymbol{q}_{n}\right\rfloor$

Where $\mathrm{q}$ is the convergence point and $\mathrm{J}$ is the robot Jacobian matrix. If the mechanism is not in a singular configuration, this expression is derived as follows [8], [9]:

$\boldsymbol{J}=\boldsymbol{J}_{x}^{-1} \boldsymbol{J}_{q}$

Where:

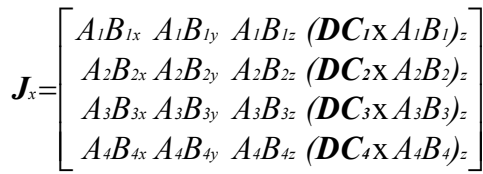

$\boldsymbol{J}_{\boldsymbol{q}}=\operatorname{diag}\left(\left(\boldsymbol{P}_{i} \boldsymbol{B}_{\boldsymbol{i}} \times \boldsymbol{A}_{\boldsymbol{i}} \boldsymbol{B}_{\boldsymbol{i}}\right) \cdot u_{m i}\right), i=1, \ldots 4$

$\boldsymbol{D} \boldsymbol{C}_{\boldsymbol{i}}$ is the distance between the center of the nacelle and the center of the half lengths of the " $\mathrm{H}$ " that forms the nacelle.

\section{B. Dynamic modelling}

In first approximation, the dynamic model is computed by considering physical dynamics. Indeed, drive torques are mainly used to move the motor inertia, the fore-arms and the arms and the nacelle equipped with a machining tool. Because of the design, the fore-arm inertia can be considered as a part of the motor inertia and the arm (manufactured in carbon materials) effects are neglected [8], [9]. 
If $\boldsymbol{\Gamma}_{\boldsymbol{m} \text { t }}$ is the $(4 \times 1)$ actuator torque vector, the basic equation of dynamics can be written as :

$$
\boldsymbol{\Gamma}_{m o t}=\mathbf{I} m o t \ddot{\mathbf{q}}+\mathbf{J}^{T} \mathbf{M}(\ddot{\mathbf{x}}-G)+\mathbf{F} v \dot{\mathbf{q}}+\mathbf{F}_{c} \operatorname{sign}(\dot{\mathbf{q}})
$$

where $\boldsymbol{I}_{\boldsymbol{m o t}}$ represents the motor's inertia matrix including the forearm's inertia, $\boldsymbol{M}$ a matrix containing the mass of the nacelle and its inertia, $\dot{\boldsymbol{q}}$ is the (4x1) joint velocity vector, $\ddot{\boldsymbol{q}}$ is $(4 \times 1)$ the joint acceleration vector, $\ddot{\boldsymbol{x}}$ is the vector of cartesian accelerations, and $\boldsymbol{G}$ the gravity constant. Thanks to the design, the forearm's inertia is taken into account in the motor's inertia. $\boldsymbol{F}_{\boldsymbol{v}}$ are the viscous friction coefficients and $\boldsymbol{F}_{\boldsymbol{c}}$ are the Coulomb friction.

With:

$\boldsymbol{I}_{m o t}=\left[\begin{array}{cccc}I_{m o t 1} & 0 & 0 & 0 \\ 0 & I_{\text {mot } 2} & 0 & 0 \\ 0 & 0 & I_{m o t 3} & 0 \\ 0 & 0 & 0 & I_{m o t 4}\end{array}\right]$

$\boldsymbol{M}=\left[\begin{array}{cccc}M n a c & 0 & 0 & 0 \\ 0 & M n a c & 0 & 0 \\ 0 & 0 & M_{n a c} & 0 \\ 0 & 0 & 0 & I_{b c}\end{array}\right]$

It is first assumed that the nacelle acceleration $\ddot{\boldsymbol{x}}=\left[\begin{array}{llll}\ddot{x} & \ddot{y} & \ddot{z} & \ddot{\theta}\end{array}\right]^{T}$ and the motor position $\boldsymbol{q}=\left[\begin{array}{llll}q_{1} & q_{2} & q_{3} & q_{4}\end{array}\right]^{T}$ are directly measured. The dynamic equation can be rewritten in a relation linear to the dynamic parameters. By introducing $\boldsymbol{J}^{T}=\left[\begin{array}{ll}\boldsymbol{J}_{43}{ }^{T}{ } \boldsymbol{J}_{41}{ }^{T}\end{array}\right]$, it follows:

$\boldsymbol{\Gamma}_{\text {mot }}=\left[\begin{array}{ll}\ddot{\boldsymbol{q}} & \boldsymbol{J}_{43}{ }^{T}\left[\begin{array}{c}\ddot{\boldsymbol{x}} \\ \ddot{y} \\ \ddot{z}-G\end{array}\right] \boldsymbol{J}_{41}{ }^{T} \ddot{\theta} \quad \dot{\boldsymbol{q}} \quad \operatorname{sigh}(\dot{\boldsymbol{q}})\end{array}\right] \boldsymbol{X}$

where $\boldsymbol{X}$ is the vector of parameters:

$$
\begin{aligned}
& \boldsymbol{X}=\left[\begin{array}{llllllll}
I_{m o t 1} & I_{m o t 2} & I_{m o t} & I_{m o t} 4 & M_{n a c} & I_{b c} \\
F_{v 1} & F_{v 2} & F_{v 3} & F_{v 4} & F_{c 1} & F_{c 2} & F_{c 3} & F_{c 4}
\end{array}\right]^{T}
\end{aligned}
$$

If acceleration measurement $\ddot{\mathbf{x}}$ is not available, $\ddot{\mathbf{x}}$ can be evaluated by:

$$
\ddot{\boldsymbol{x}}=\boldsymbol{J} \ddot{\boldsymbol{q}}+\dot{\boldsymbol{J}} \dot{\boldsymbol{q}}=\left[\begin{array}{llll}
\ddot{x}_{q} & \ddot{y}_{q} & \ddot{z}_{q} & \ddot{\theta}_{q}
\end{array}\right]^{T}
$$

where $\boldsymbol{J}$ depends on $\boldsymbol{x}$ and $\boldsymbol{q}, \boldsymbol{J}$ is computed using a central difference algorithm.

Then, the second identification dynamic model is given by :

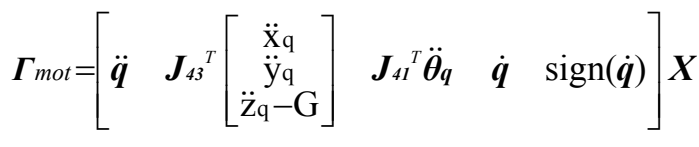

Both models are expressed in a general form:

$\boldsymbol{Y}=\boldsymbol{D} \boldsymbol{X}$

where $\boldsymbol{Y}$ is the torque measurement vector, $\boldsymbol{D}$ is called the regressor and $\boldsymbol{X}$ is the vector of unknown parameters.

\section{IDENTIFICATION METHOD}

The identification technique, classically developed for robot manipulators, is applied for the parallel robot. Usually $\boldsymbol{X}$ is estimated as the least squares (LS) solution of an overdetermined linear system obtained by sampling and filtering the dynamic model (20) along a trajectory $(\boldsymbol{q}, \dot{\boldsymbol{q}}, \ddot{\boldsymbol{q}})$, considering that $\boldsymbol{\rho}$ is a zero mean additive independent noise, with a standard deviation $\sigma_{\rho}$ such that:

$\boldsymbol{C}_{\boldsymbol{\rho} \boldsymbol{\rho}}=E\left(\boldsymbol{\rho}^{\boldsymbol{T} \rho}\right)=\sigma_{\rho}^{2} \boldsymbol{I}_{r}$

where $E$ is the expectation operator. $\boldsymbol{I}_{\boldsymbol{r}}$ is the (rxr) identity matrix. The over-determined system is written as follows:

$\boldsymbol{Y}=\boldsymbol{W} \boldsymbol{X}+\boldsymbol{\rho}$

where $\boldsymbol{Y}$ is the (rx1) measurement vector, $\boldsymbol{W}$ is the $(r \times N)$ observation matrix, $N$ is the number of parameters to identify. In fact $\boldsymbol{Y}$ is obtained by concatenation of $\mathrm{n}$ measurements vectors $\boldsymbol{Y}^{j}$ of the $n$ motor torques with different error standard deviations. A better solution is to calculate the WLS solution of the global system (22). The $r^{j}$ rows, corresponding to joint $j$ equation, are weighted by the coefficient of the diagonal matrix of the error covariance matrix defined as follows:

$\mathbf{C}_{\boldsymbol{\rho} \rho}=\left(\boldsymbol{G}^{\boldsymbol{T}} \boldsymbol{G}\right)^{-1} \quad \boldsymbol{G}=\operatorname{diag}(\boldsymbol{S})$

$\boldsymbol{G}$ is a $(r \times r)$ diagonal matrix composed of the elements of $\boldsymbol{S}$.

$\boldsymbol{S}=\left[S^{l} \ldots S^{n}\right], \boldsymbol{S}^{j}=\left[\frac{1}{\hat{\sigma}^{j}} \ldots \frac{1}{\hat{\sigma}^{j}}\right]$

$S^{j}$ is a $\left(l \times r^{j}\right)$ row matrix. An unbiased estimation $\hat{\sigma}_{\rho}^{j}$ is used from the regression on each joint $j$ subsystem:

$\hat{\sigma} \rho^{j}=\frac{\left\|Y^{j}-\Phi^{j} \hat{\Theta}^{j}\right\|^{2}}{\left(r^{j}-N_{p}^{j}\right)}$

$\boldsymbol{Y}^{j}, \boldsymbol{\Phi}^{j}, \Theta^{j}, \boldsymbol{r}^{j}, \boldsymbol{N p}^{j}$ are the measurement vector, the observation matrix, the number of equations and the number 
of minimum parameters for each joint $j$ subsystem respectively.

The WLS vector solution $\hat{\boldsymbol{X}}_{\boldsymbol{w}}$ minimizes the Euclidean norm of the vector of weighted errors $\rho$ :

$$
\hat{\boldsymbol{X}}_{\boldsymbol{w}}=\underset{X}{\operatorname{Arg} . \min }\left\lfloor\rho^{T} G^{T} G \rho\right\rfloor
$$

$\hat{\boldsymbol{X}}_{\boldsymbol{w}}$ and the corresponding standard deviations $\sigma_{\hat{X}_{w i}}$ are calculated as the LS solution of (22) weighted by $\boldsymbol{G}$ :

$\boldsymbol{Y}_{w}=\boldsymbol{W}_{w} \boldsymbol{X}+\boldsymbol{\rho}_{w}$

$\boldsymbol{Y}_{w}=\boldsymbol{G} \boldsymbol{Y}, \boldsymbol{W}_{w}=\boldsymbol{G} \boldsymbol{W}, \boldsymbol{\rho}_{w}=\boldsymbol{G} \boldsymbol{\rho}$

Complete details concerning the WLS identification technique and its practical implementation can be found in [10], [11], [12].

\section{EXPERIMENTAL RESULTS}

\section{A. Determination of the amplifier gain}

For industrial robots, torques are usually estimated using a linear relation between torque and voltage applied to the amplifier:

$$
\Gamma_{m}=G_{T} V_{T}
$$

where $V_{T}$ is the current reference of the amplifier current loop and $G_{T}$ the gain of the joint drive chain. A good estimation of $G_{T}$ is important to obtain a good estimation of the physical parameters.

A force sensor, located on the nacelle, is used to measure directly the force produced at the arm end. Applying different input tensions to the amplifier, the resulting torques are measured and the gain is estimated (Table 1). In [10], other techniques to estimate the $G_{T}$ values are given.

\section{B. Dynamic parameters estimation}

In this work, we are focused in the estimation of the following dynamic parameters:

$$
\begin{aligned}
& \boldsymbol{X}=\left[\begin{array}{llllllll}
I_{m o t} 1 & I_{m o t} 2 & I_{m o t} & I_{m o t} 4 & M_{n a c} & I_{b c} \\
F_{v 1} & F_{v 2} & F_{v 3} & F_{v 4} & F_{c 1} & F_{c 2} & F_{c 3} & F_{c 4}
\end{array}\right]
\end{aligned}
$$

TABLE 1

AMPLIFIERS GAINS

\begin{tabular}{|l|c|c|c|c|}
\hline & Motor 1 & Motor 2 & Motor 3 & Motor 4 \\
\hline$G_{T}(N . m / V)$ & 2.85 & 2.65 & 2.70 & 2.87 \\
\hline
\end{tabular}

For computing the regressor, joint velocities and accelerations are estimated by a band pass filtering of the position. The band pass filtering is obtained by the product of a low pass filter in both the forward and the reverse direction (Butterworth) and a derivative filter obtained by a central difference algorithm, without phase shift. A parallel filtering is implemented to reject the high frequency ripples of the measured motor torques. Practical aspects of the derivative estimation and data filtering are completely detailed in [13] and [14].

In order to get good identification results, exciting trajectories containing slow motions (in such a case, friction will be preponderant) and high dynamic motions (inertia phenomena become preponderant) are generated. Finally, concatenation of these trajectories is used. Examples of generated trajectories are presented in Fig. 3.

1) Identification without additional sensor: Initially, the Cartesian accelerations are computed thanks (18), where the actuator velocities and accelerations have been computed from the actuators positions. Table 2 shows the obtained parameters of the dynamics model (19). The relative standard deviation $\left(\% \sigma_{\hat{x} r}\right)$ is given.

The physical parameters are quite well estimated in comparison to the prior values of nacelle mass and the motor inertia $\left(0.975 \mathrm{Kg}\right.$ and $0.012 \mathrm{~N} . \mathrm{m}^{2}$ respectively). However additional measurements are provided to improve estimation accuracy.

2) Identification using additional sensors: An accelerometer located on the nacelle gives the Cartesian accelerations on the
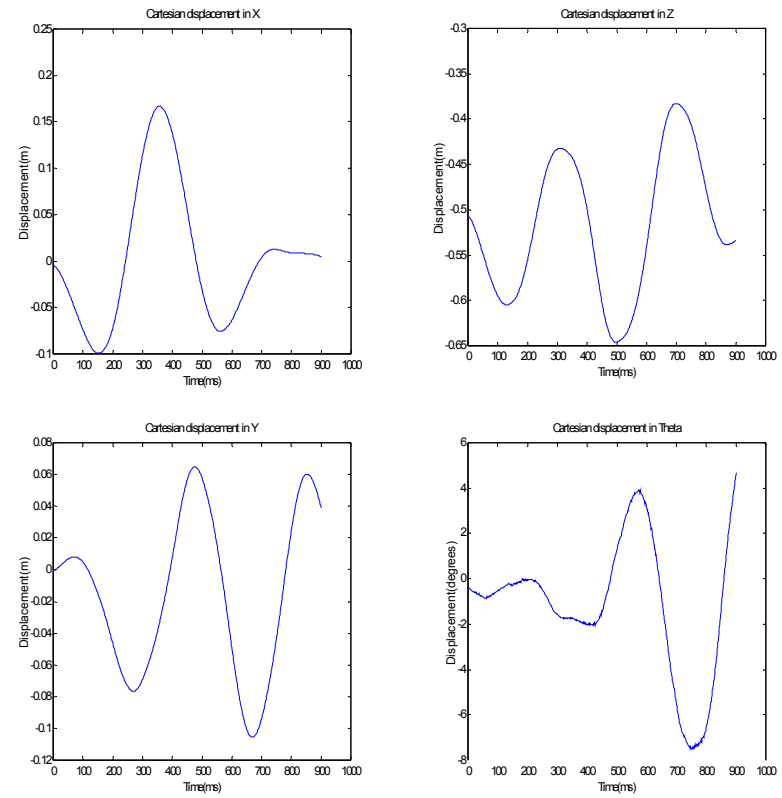

Fig. 3 Typical trajectories in the Cartesian space 
TABLE 2

\begin{tabular}{|c|c|c|c|}
\hline Parameter & $\begin{array}{c}\text { Estimated } \\
\text { values }\end{array}$ & Units & $\% \sigma_{\hat{x} r}$ \\
\hline$I_{m o t 1}$ & 0.0141 & N.m ${ }^{2}$ & 2.6286 \\
\hline$I_{\text {mot } 2}$ & 0.0120 & N.m ${ }^{2}$ & 3.0444 \\
\hline$I_{m o t 3}$ & 0.0153 & N.m ${ }^{2}$ & 1.6939 \\
\hline$I_{m o t 4}$ & 0.0213 & N.m ${ }^{2}$ & 1.1933 \\
\hline$M_{n a c}$ & 1.0492 & $\mathrm{Kg}$ & 0.4236 \\
\hline$I_{b c}$ & 0.0030 & N.m ${ }^{2}$ & 3.5049 \\
\hline$F_{v 1}$ & 0.1636 & N.m.s/rad & 5.6781 \\
\hline$F_{v 2}$ & 0.0560 & N.m.s/rad & 15.5674 \\
\hline$F_{v 3}$ & 0.0930 & N.m.s/rad & 6.5734 \\
\hline$F_{v 4}$ & 0.0917 & N.m.s/rad & 6.4301 \\
\hline$F_{c 1}$ & 1.1453 & N.m & 2.0450 \\
\hline$F_{c 2}$ & 1.0950 & N.m & 2.0563 \\
\hline$F_{c 3}$ & 0.7222 & N.m & 2.8366 \\
\hline$F_{c 4}$ & 0.9932 & N.m & 2.0451 \\
\hline
\end{tabular}

three axes $(x, y$ and $z$ ) and a rotation sensor gives the central bar rotation. We use them to perform the identification straight with the model of (19). Fig. 4 shows the comparison of measured data obtained from the accelerometer and rotation sensor and those provided by (16). Rotation acceleration is numerically computed by a finite central difference.

Table 3 shows the fourteen estimated parameters of the dynamic model (16). In case of the rigid multi body model identification, additional sensors located on the end effector mainly improve the estimation of the viscous friction coefficients and their relative standard deviations.

\section{Experimental validation}

The validation of the identification results consists in comparing the measured torques with those obtained by computing the inverse dynamic model with the estimated parameters. Fig. 5 exhibits cross validations with new trajectories that have not been used previously for the identification. Simulation and measurements are very close. The dynamic parameters are quite well estimated.

These experiments show good results of the dynamic identification. The use of the accelerometer and rotation sensor are not very necessary for the rigid model.

\section{CONCLUSION}

In this paper experimental results related to the identification of physical dynamic parameters of a fully parallel robot are presented. Estimated values depending on the available measurements at the end effector are exhibited. The cross validation shows good identification results.
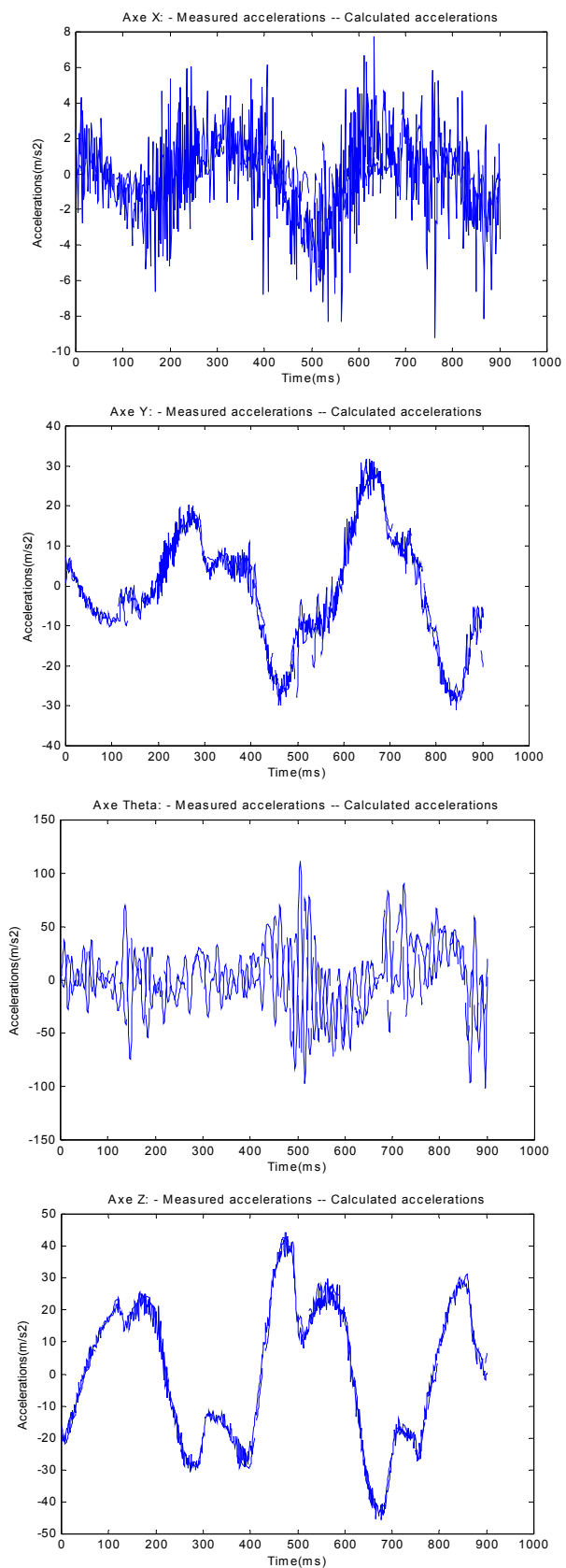

Fig. 4 Calculated and measured accelerations

However this structure working with high speed and acceleration presents important flexibility and slight differences during validation may be due to flexibility. Therefore, we are currently working on the introduction of lumped elasticities in the dynamic model. In the future, we will compare the results with other estimation methods and this complete dynamic model will be used in model based control scheme with moving horizon for machining tasks. 
TABLE 3

ESTIMATED PARAMETERS USING ADDITIONAL SENSORS

\begin{tabular}{|l|r|r|r|}
\hline \multicolumn{1}{|c|}{ Parameter } & $\begin{array}{c}\text { Estimated } \\
\text { values }\end{array}$ & \multicolumn{1}{c|}{ Units } & \multicolumn{1}{c|}{$\% \sigma_{\hat{x} r}$} \\
\hline$I_{m o t 1}$ & 0.0167 & N.m & 2.3695 \\
\hline$I_{m o t 2}$ & 0.0164 & N.m $^{2}$ & 2.3590 \\
\hline$I_{m o t 3}$ & 0.0176 & N.m $^{2}$ & 1.5776 \\
\hline$I_{m o t 4}$ & 0.0234 & $\mathrm{~N}^{2}$ & 1.1579 \\
\hline$M_{n a c}$ & 0.984 & $\mathrm{Kg}$ & 0.4666 \\
\hline$I_{b c}$ & 0.0029 & $\mathrm{~N} . \mathrm{m}^{2}$ & 3.7311 \\
\hline$F_{v 1}$ & 0.2112 & N.m.s $/ \mathrm{rad}$ & 4.7212 \\
\hline$F_{v 2}$ & 0.1236 & N.m.s/rad & 7.5670 \\
\hline$F_{v 3}$ & 0.1266 & N.m.s $/ \mathrm{rad}$ & 5.2000 \\
\hline$F_{v 4}$ & 0.1133 & N.m.s/rad & 5.6255 \\
\hline$F_{c 1}$ & 1.2186 & N.m & 2.0756 \\
\hline$F_{c 2}$ & 1.0252 & N.m & 2.3623 \\
\hline$F_{c 3}$ & 0.7902 & N.m & 2.7986 \\
\hline$F_{c 4}$ & 1.0394 & N.m & 2.1046 \\
\hline
\end{tabular}

\section{REFERENCES}

[1] V.E. Gough, "Contribution to discussion of papers on research in automotive stability, control and tyre performance", Proc. Auto Div., Inst. Mechanical Engineers, 1956-1957.

[2] D. Stewart, "A plataform with 6 degrees of freedom", Proc. of the Ins. of Mech. Engineers, 180 (Part 1, 15), pp. 371-386, 1965.

[3] R. Clavel, "Une nouvelle structure de manipulateur parallèle pour la robotique légère", APII, 23 (6), pp. 501519, 1989.

[4] J.-P. Merlet, Les robots parallèles, $2^{\text {nd }}$ Edition, Hermes, 1997.

[5] H. K. Tönshoff, "A systematic comparison of parallel kinematics", Keynote in proceedings of the first forum on parallel kinematic machines, Milan, Italy, August 31 September 1, 1998.

[6] F. Pierrot, T. Shibukawa, "From Hexa to HexaM", Proc. IPK'98: Internationales Parallelkinematik-Kolloquium, Zürich, June 4, pp. 75-84, 1998.

[7] F. Pierrot, P. Dauchez and A. Fournier, "Fast parallel robots", Journal of robotics systems, 8 (6), pp. 829-840, 1991.

[8] O. Company, F. Pierrot, "A new 3T-1R parallel robot", ICAR'99, Tokyo, Japan, October 25-27, pp. 557-562, 1999.

[9] F. Pierrot, F. Marquet, O. Company, T. Gil, "H4 Parallel Robot,: Modeling, Design and Preliminary Experiments", Proc. of the 2001 IEEE International Conference one Robotics \& Automation, Seoul, Korea, May 21-26, 2001.

[10] P. Restrepo, "Contribution à la modélisation, identification et commande des robots à structures fermées: application au robot ACMA SR400", Ph.D Thesis, Ecole Centrale de Nantes, France, 1996.

[11] Ph. Poignet, M. Gautier, "Extended kalman filtering and weighted least squares dynamic identification of robots", Control Engineering Practice, 2001, vol. 9/12, pp. 1361-
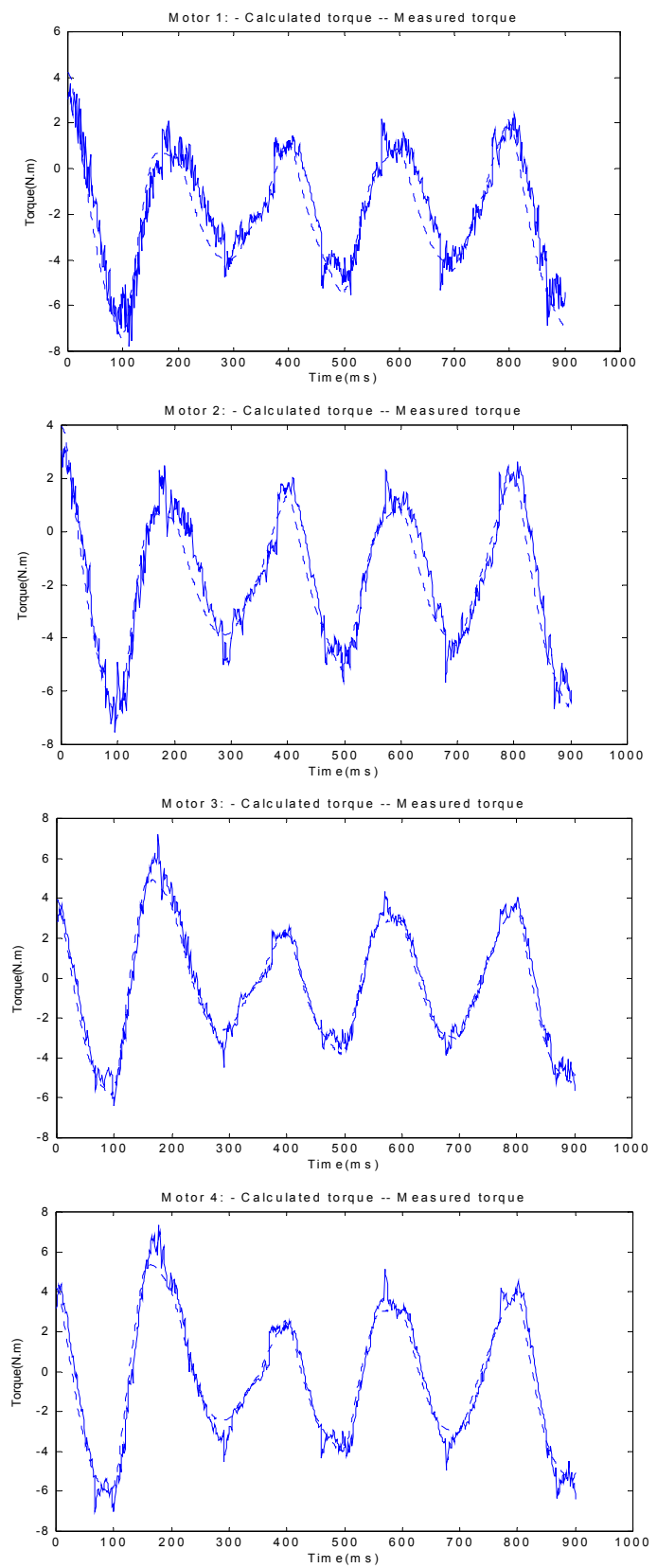

1372.

Fig. 5 Calculated and measured torques

[12] C. Canudas de Wit, B. Siciliano, G. Bastin, Theory of robot control, Springer, 1996.

[13] W. Khalil, E. Dombre, Modeling, Identification and Control of Robots, Hermes Penton Science, London, 2002.

[14] M.T. Pham, M. Gautier, Ph. Poignet, "Dynamic identification of high speed machine tools", Proc. of the II International Seminar on Improving Machine Tools Performance, La Baule, France, 2000. 